\title{
Indirect detection of composite asymmetric dark matter
}

\author{
Rakhi Mahbubani॰* \\ Albert Einstein Center for Fundamental Physics, Institute for Theoretical Physics, \\ University of Bern, Sidlerstrasse 5, CH-3012 Bern, Switzerland \\ and Theoretical Physics Department, CERN, 1211 Geneva 23, Switzerland \\ Michele Redi $\odot^{\dagger}$ and Andrea Tesi $\odot^{*}$ \\ ${ }^{2}$ INFN Sezione di Firenze, Via G. Sansone 1, I-50019 Sesto Fiorentino, Italy \\ and Department of Physics and Astronomy, University of Florence, Italy
}

(Received 12 August 2019; accepted 7 May 2020; published 27 May 2020)

\begin{abstract}
Dark matter can form bound states upon the emission of quanta of energy equal to the binding energy. The rate of this process is large for strongly interacting dark matter, and further enhanced by long-distance effects. The resulting monochromatic and diffuse $\gamma$ rays can be tested in indirect-detection experiments. If dark matter has electroweak charge, indirect signals include multiple observable photon lines for masses in the $\mathrm{TeV}$ range. Otherwise, if it couples only via a dark photon portal, diffuse spectra from dwarf galaxies and cosmic microwave background reionization set powerful limits for masses below a tera-electron volt. This mechanism provides a powerful means of probing asymmetric dark matter today.
\end{abstract}

DOI: 10.1103/PhysRevD.101.103037

\section{INTRODUCTION}

Having no annihilation rate today, asymmetric dark matter (DM) is largely untestable in indirect-detection experiments absent some nonminimal assumption (e.g., a remnant annihilating component, or decaying relic, see $[1,2]$ for reviews on the subject). We argue in a compelling analogy with Standard Model (SM) baryons that "nucleons" of a dark strong sector naturally emit a light particle on forming bound-state "nuclei." The rate for this process is calculable semianalytically in the limit of shallow bound states, and can be large, allowing us to probe dark nuclear asymmetric DM in existing indirect-detection experiments. (See [3-7] for related work).

While our considerations apply more generally, e.g., to conventional thermal DM, we focus here on models where $\mathrm{DM}$ is asymmetric, and composite due to dark strong interactions, in close analogy with SM nucleons. The thermal abundance of DM is reproduced for masses around $100 \mathrm{TeV}$ [8] so that for masses below this value the symmetric component of DM is subleading. Composite DM can be simply realized as the lightest baryon in an

\footnotetext{
rakhi@cern.ch

†michele.redi@fi.infn.it

\$andrea.tesi@fi.infn.it
}

Published by the American Physical Society under the terms of the Creative Commons Attribution 4.0 International license. Further distribution of this work must maintain attribution to the author(s) and the published article's title, journal citation, and DOI. Funded by SCOAP.
$\mathrm{SU}(N)$ confining gauge theory with dark fermions that are vectorial under the SM [8]; see [9] for a review. DM cosmological stability follows from the accidental dark baryon-number conservation, which also guarantees the stability of the lightest state in each baryonic sector. The dark sector is roughly characterized at low energies by (i) the mass of the lightest dark baryon, $M$, which constitutes the DM; (ii) the mass of the "dark pion," $M_{\pi} \lesssim M$, that sets the typical range for nuclear interactions amongst the baryons (we assume that this state is cosmologically unstable); and (iii) the mass of a weakly coupled mediator external to the strong sector, $M_{V}$, e.g., SM gauge bosons. We will assume that the spectrum features nuclear bound states with binding energies $E_{B}>M_{V}$, focusing in particular on the nucleus with baryon number 2, "dark deuterium."

The cosmological production of dark nuclei was studied in [10]; here we consider observational implications. At DM velocities relevant for indirect detection, dark deuterium is produced essentially at rest through emission of a quantum of energy $E_{B}$. The process is depicted in Fig. 1.

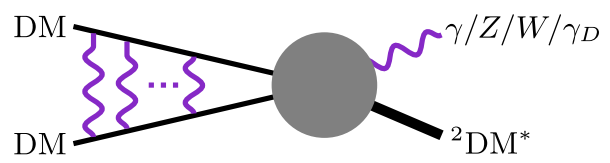

FIG. 1. Bound-state formation considered in this paper. The process is affected by (long-) short-distance ("weak") "nuclear" physics. The bound state can be unstable and decay to the ground state emitting additional lines. 
We will consider two main scenarios, characterized by the properties of the mediator carrying the quantum of energy emitted in bound-state formation. The first is automatically realized if DM has electroweak charges, and gives rise to emission of SM gauge bosons, in particular, to monochromatic photons. These are constrained by observations of the Galactic Center by FERMI and HESS. In the second scenario, DM is neutral under the SM but charged under an additional (broken) U(1). Dark deuterium is then produced in association with a dark photon that later decays to SM particles. The resulting diffuse photon signal can be tested using observations of dwarf spheroidal galaxies and cosmic microwave background (CMB) reionization. As we will discuss shortly, both alternatives can be analyzed by exploiting the analogy with deuterium production in the $\mathrm{SM}$, yet they present very different experimental signatures.

\section{NUCLEAR CROSS SECTIONS}

We consider the production of shallow nuclear bound states with $E_{B} \ll M$. As shown by Bethe and Longmire [11], and more recently derived systematically using nucleon effective field theories [12], at low energy the cross section for the formation of a shallow bound state does not depend on the details of the potential, but simply on the parameters of the effective range expansion. The amplitude for elastic scattering is determined by the phase shift $\delta$, which for $s$-wave scattering admits the following expansion:

$$
\begin{aligned}
\mathcal{A} & =\frac{4 \pi}{M} \frac{1}{p \cot \delta-i p}, \\
p \cot \delta & =-\frac{1}{a}+\frac{1}{2} r_{0} p^{2}+\ldots,
\end{aligned}
$$

where $p$ is the momentum of the incoming states in the center-of-mass frame, $a$ is the scattering length, and $r_{0}$ is the range of the interaction, $r_{0} \sim 1 / M_{\pi}$. Importantly for a shallow $s$-wave bound state, the scattering length is determined by the binding energy, $1 / a \approx \sqrt{E_{B} M}$, as can be seen from the pole of $\mathcal{A}$. The deuteron formation cross section is computed in terms of the binding energies/ scattering lengths of the $n p$ elastic scattering channels ${ }^{1} S_{0}$ and ${ }^{3} S_{1}$ [13]. Indeed, in the presence of bound states (i.e., poles of the elastic amplitude for imaginary momenta $p$ ), the elastic scattering amplitude allows one to extract the coupling of the deuteron to two nucleons from the residue at the pole and calculate the cross section with Feynman diagrams; see [14] for a review.

In this paper we focus on dark nuclear transitions induced by magnetic-dipole interactions,

$$
\kappa \frac{e}{M} N^{\dagger} J_{3}(\vec{\sigma} \cdot \vec{B}) N,
$$

where $N$ is the nonrelativistic dark nucleon field, $J_{3}$ is the third component of isospin, and $\kappa \sim 1$ for strongly coupled nucleons. This interaction induces transitions with selection rules $\Delta L=0$ and $\Delta S=1$, allowing for bound-state formation from an initial $s$-wave state. Nuclei can also be produced through electric dipole transitions but this process is typically suppressed at low velocities; see Ref. [15].

The cross section for the formation of an $s$-wave bound state through a magnetic transition reads [10]

$$
\left(\sigma v_{\mathrm{rel}}\right)_{N N \rightarrow D \gamma}^{\mathrm{mag}} \approx \kappa^{2} K_{M} \frac{4 \pi \alpha}{M^{2}}\left(\frac{E_{B_{f}}}{M}\right)^{\frac{3}{2}}\left(1-\frac{a_{i}}{a_{f}}\right)^{2},
$$

where $a_{i, f}$ are the scattering lengths of initial and final state, $v_{\text {rel }}$ is the relative velocity of the incoming states in the center-of-mass frame, and $K_{M}$ is a group theory factor, equal to 1 for deuteron formation in the SM.

This cross section can be significantly modified by longdistance effects due to forces external to the strong sector [16]. Such long-distance modification is intimately tied to the mechanism of bound-state formation, which can only take place through emission of the light quanta that are responsible for the effect. For electroweak constituents these forces are just SM gauge interactions, while for SMneutral constituents we consider the possibility that they are associated with the exchange of a dark photon. In the case of DM annihilation, the long-distance effects can be factorized so that $\sigma \approx \mathrm{SE} \times \sigma_{\text {short }}$, where $\mathrm{SE}$ is the Sommerfeld enhancement factor that takes into account the distortion of the initial wave function due to long-range forces and $\sigma_{\text {short }}$ is the perturbative cross section. As we will discuss in detail in [15], for bound-state formation the longdistance effects often cannot be simply factorized. As a result, in this paper we carry out a full quantum-mechanical computation of the cross section by the explicit solution of the Schrödinger equation to obtain the physical wave function; see [17].

The reduced wave function describing $s$-wave scattering of two DM particles of mass $M$, in a given spin/charge sector, $u(r)=\sqrt{4 \pi} r \psi(r)$ satisfies the radial Schrödinger equation,

$$
-\frac{1}{M} \frac{d^{2} u}{d r^{2}}+V(r) u=E u,
$$

where $E=M \beta^{2}$ for $\beta=v_{\text {rel }} / 2$. The wave function $u(r)$ is in general a vector, on which we impose physical boundary conditions: $u(0)=0, u^{\prime}\left(r_{\infty}\right)-i p u\left(r_{\infty}\right)=\sqrt{4 \pi} e^{-i p r_{\infty}} u_{0}$ where $u_{0}$ denotes the DM initial state.

The potential $V(r)$, defined in a given spin/charge sector, contains a long-distance part, associated for example with electroweak interactions, as well as a short-distance, spherically symmetric nuclear potential $V_{N}$ that respects the flavor symmetry of the strong dynamics. To leading order the nuclear potential must simply reproduce the correct binding energies and range of interaction. We choose to parametrize it using a spherical well in each irreducible representation $a$ of the global symmetry of the nuclear 
interactions, $V_{a}^{N}=-V_{a} \theta\left(r_{0}-r\right)$. The depth of the well $V_{a}$ determines the binding energy, which we select in order to have a single shallow bound state per channel [18]. The reduced wave function describing the corresponding bound state is known analytically in the isospin-symmetric limit, and is given roughly by $u_{f}(r) \approx$ $\left(4 M E_{B_{f}}\right)^{\frac{1}{4}} \exp \left(-\sqrt{M E_{B_{f}}} r\right)$. The magnetic cross section can then be computed as follows:

$$
\left(\sigma v_{\mathrm{rel}}\right)^{\mathrm{mag}}=8 \kappa^{2} \alpha \frac{E_{B_{f}}^{3}}{M^{2}} \times\left|\int d r u_{i}^{\dagger} J_{3} u_{f}\right|^{2},
$$

where $u_{i}$ and $u_{f}$ are the reduced wave functions of initial and final states. We take $\kappa=1$ for the remainder of this paper.

\section{COMPOSITE SU(2)-TRIPLET DM}

We consider a scenario with a fermionic dark nucleon $V$ that transforms as a triplet of $\mathrm{SU}(2)_{L}$. This can be realized in an $\mathrm{SU}(3)$ dark gauge theory with three flavors [8]. Like for the wino, electroweak symmetry-breaking effects induce a mass splitting $\Delta=165 \mathrm{MeV}$ between the charged $V_{ \pm}$and neutral $V_{0}$ components. Collider bounds due to dark pion production require $M \gtrsim \mathrm{TeV}$.

The nuclear potential being $\mathrm{SU}(2)_{L}$ symmetric, all composite states can be classified according to their spin and weak isospin in each partial wave. The lightest dark nuclei (isotopes of dark deuterium) are $s$-wave bound states of two dark nucleons $V$, with isospin-spin $3 \times 3=$ $1_{0}+3_{1}+5_{0}$; we name them $D_{1}, D_{3}$, and $D_{5}$ respectively. The selection rules of the magnetic-dipole operator in Eq. (2) allow for $s$-wave transitions in isospin channels $1_{0} \leftrightarrow 3_{1}$ and $3_{1} \leftrightarrow 5_{0}$. Cosmological production of bound states being typically small for $\mathrm{DM}$ masses in the $\mathrm{TeV}$ range [10], we will take DM today to be composed entirely of neutral nucleons, $V_{0}$. The antisymmetry of its wave function implies that an $s$-wave initial state must have spin 0 . The magnetic-dipole transition then allows for the production of an $s$-wave spin-1 nuclear bound state, the neutral component of the $\mathrm{SU}(2)_{L}$ triplet, $D_{3}^{0}$. Including for simplicity only the singlet nuclear potential and electroweak interactions, the potential in the charge- 0 spin- 0 subsector containing $V_{+} V_{-}$and $V_{0} V_{0}$ reads

$V_{Q=0}^{S=0}=\left(\begin{array}{cc}2 \Delta-A & -\sqrt{2} B \\ -\sqrt{2} B & 0\end{array}\right)+V_{1}^{N}(r)\left(\begin{array}{cc}\frac{2}{3} & \frac{\sqrt{2}}{3} \\ \frac{\sqrt{2}}{3} & \frac{1}{3}\end{array}\right)$,

where $A=\alpha / r+\alpha_{2} c_{W}^{2} e^{-M_{Z} r} / r, B=\alpha_{2} e^{-M_{W} r} / r$ are the usual electroweak contributions while $V_{I}^{N}(r)$ is the nuclear potential in the isospin- $I$ channel, rotated to the charge basis. In the spin-1 channel we neglect small corrections due to electroweak effects and take $V_{Q=0}^{S=1}=V_{3}^{N}(r)$.

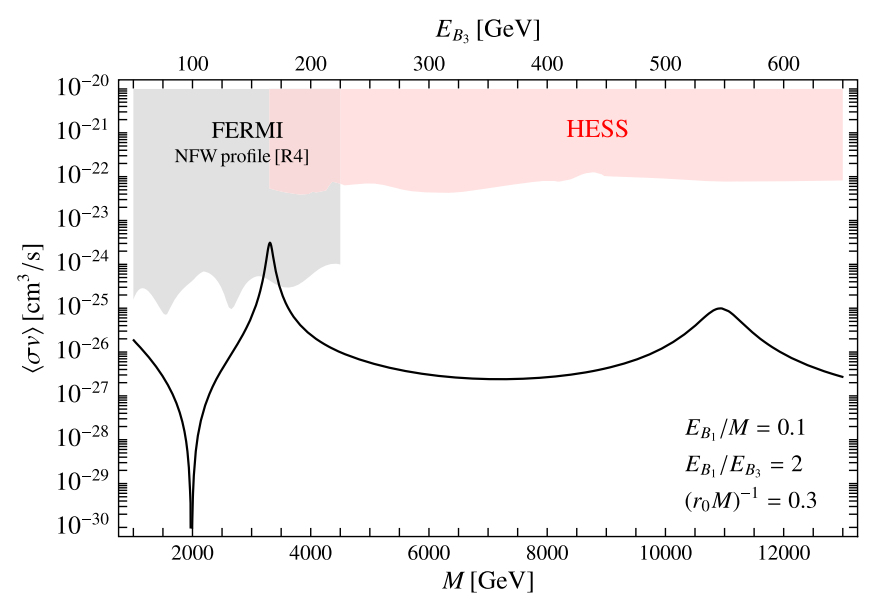

FIG. 2. Bound-state production cross section for a composite fermion triplet of SU(2) by photon emission, $V_{0} V_{0} \rightarrow D_{3}^{0} \gamma$. We assume negligible potential in the quintuplet channel. The gray (red) region is the exclusion due to $\gamma$-ray lines from FERMI [19] (HESS [20]) in our Galactic Center.

In Fig. 2 we report the cross section for the production of $D_{3}^{0}$ by emission of a photon, and compare with experimental constraints on $\gamma$-ray line spectra in the Galactic Center due to FERMI [19] and HESS (as extracted from [20]). In making the comparison we need to account for the reduced energy of a photon coming from bound-state formation, as compared with that from direct annihilation of DM to photons; while the DM number density is determined by $M$, the energy emitted is $E_{B} \ll M$. Thus, to extract experimental bounds the limit on the annihilation cross section must be rescaled as follows:

$$
\left\langle\sigma_{D \gamma} v_{\text {rel }}\right\rangle<2\left(\frac{M}{E_{B}}\right)^{2}\left\langle\sigma_{\gamma \gamma} v_{\text {rel }}\right\rangle_{M_{\mathrm{DM}}=E_{B}}
$$

where the factor of 2 is due to the emission of a single photon in bound-state formation.

For a conservative choice of parameters, formation of dark deuterium produces a signal just within FERMI sensitivity for masses around 3-4 TeV, where the cross section is significantly enhanced due to the presence of a virtual zero-energy resonance in the initial state channel. Note that the position of the peak is slightly shifted with respect to that seen in the annihilation of wino DM due to the strong nuclear potential, which induces a shift in the binding energy of the shallow bound state. The dip in the cross section around $2 \mathrm{TeV}$ can be explained, in the nucleon effective field theory, as a destructive interference between the $V_{0} V_{0} \rightarrow D_{3}^{0}+\gamma$ and $V_{0} V_{0} \rightarrow\left(V_{+} V_{-}\right)^{*} \rightarrow D_{3}^{0}+\gamma$ diagrams. More details will be given in [15].

Note that the bound state formed, $D_{3}^{0}$, is not generically the ground state; the SM electroweak interactions, and likely also the strong interactions, favor the spin- 0 singlet $D_{1}^{0}$ to be lightest isotope. This implies that the triplet will 
subsequently decay to the ground state through a magnetic transition with rate $\Gamma \sim \kappa^{2} \alpha \sqrt{E_{B_{1}} E_{B_{3}}}\left(E_{B_{1}}-E_{B_{3}}\right)^{2} / M^{2}$ [10], leading to a second monochromatic photon signal with energy $E_{\gamma}=E_{B_{1}}-E_{B_{3}}$. Multiple photon lines with equal rate would be a smoking gun signature for boundstate formation in the dark sector, allowing us to easily discriminate it from signals due to DM annihilation.

\section{COMPOSITE SM-SINGLET DM}

In models with SM-singlet nucleons, formation of heavier nuclei requires interaction with a (SM-neutral) light state to carry away the binding energy emitted in the process. This state can be a dark photon. We assume that DM belongs to an asymmetric dark baryon doublet, $\left(N_{+}, N_{-}\right)$with equal mass and opposite dark photon charge. This can be realized with a confining SU(3) dark gauge theory with two degenerate flavors with opposite unit charges. More general assignments are possible.

The nuclear potential has a flavor-singlet spin-1 channel, and a flavor-triplet spin- 0 channel. Including the dark photon interaction, the potential in the neutral sector is described by

$$
V_{Q=0}^{S=0,1}=-\alpha_{D} \frac{e^{-M_{V} r}}{r}+V_{3,1}^{N}(r)
$$

We neglect charged channels, which are repulsive and lead to smaller cross sections.

Through a magnetic transition $N_{+}$and $N_{-}$can form bound states with spin-0 (1), with the emission of a dark photon with energy $E_{B_{3}}\left(E_{B_{1}}\right)$ respectively. The cross section for the process $N_{+} N_{-} \rightarrow D_{1,3}+\gamma_{D}$ can be computed using (5) with an extra factor of $1 / 4$ to account for distinguishable particles in the initial state. The dark photon then decays to the SM through kinetic mixing between the dark and hypercharge field strengths,

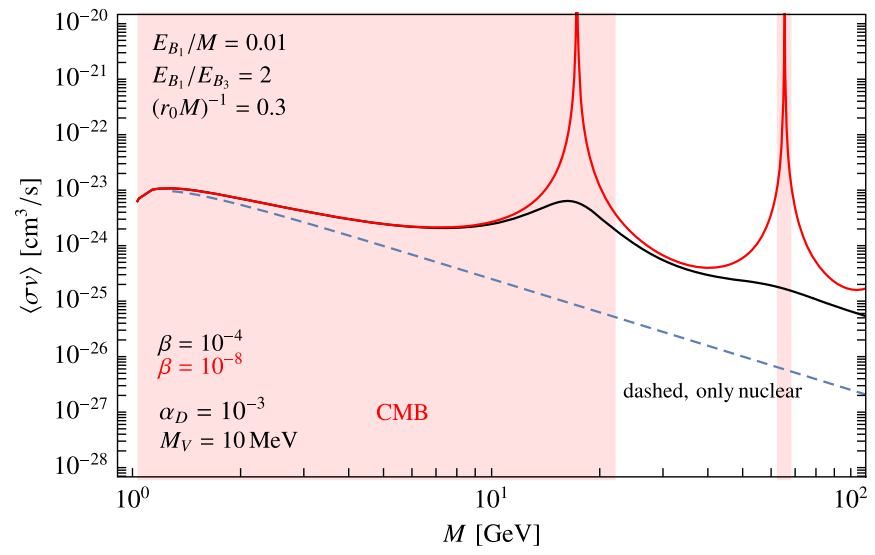

$$
\mathcal{L}_{D}=-\frac{1}{4} F_{D \mu \nu} F_{D}^{\mu \nu}-\frac{1}{2} M_{V}^{2} V_{\mu} V^{\mu}-\frac{\epsilon}{2 c_{W}} F_{D \mu \nu} B^{\mu \nu}
$$

The dark photon phenomenology is similar to that of weakly coupled models; see, for example, [21] for the allowed region of the $\left(\epsilon, M_{V}\right)$ parameter space.

In order to estimate the indirect-detection bounds due to diffuse photons, we recast the analysis of [22]. If DM is light, bound-state formation is also strongly limited by hydrogen reionization, since the CMB provides a rather model-independent bound on the energy injected into the thermal photon bath after recombination [23]. By using the result from PLANCK [24], this bound translates in our case to

$\left\langle\sigma_{D \gamma^{*}} v_{\text {rel }}\right\rangle_{\mathrm{CMB}}<\frac{8.2 \times 10^{-28} \mathrm{~cm}^{3} \mathrm{~s}^{-1}}{f_{\text {eff }}} \times \frac{M^{2}}{E_{B}^{2}} \times \frac{E_{B}}{\mathrm{GeV}}$,

where the efficiency factor $f_{\text {eff }}$ depends mildly on the decay channel. For our purposes we will take $f_{\text {eff }} \approx 0.5$. Neglecting long-distance effects (that tend to increase the cross section), the nuclear rate in Eq. (3) indicates that binding energies have to be relatively small in order to satisfy the strong CMB bounds, namely, $E_{B} / M \lesssim$ $10^{-3}(M / \mathrm{GeV})^{6 / 5}\left(0.001 / \alpha_{D}\right)^{2 / 5}$.

Estimates of the bounds on this scenario from dwarf spheroidal galaxies and the CMB are shown in Fig. 3 with the latter always yielding the stronger constraint. The strongest bound arises from the formation of $D_{1}$ which is enhanced with respect to $D_{3}$ production by a relative factor of $\left(E_{B_{1}} / E_{B_{3}}\right)^{5 / 2}$. The limit from dwarf spheroidals is sensitive to the dark photon branching fraction, and could vary by a factor of 5 in either direction for a different choice of mass. For our specific choice of parameters, we have verified that the cosmological production of dark deuterium

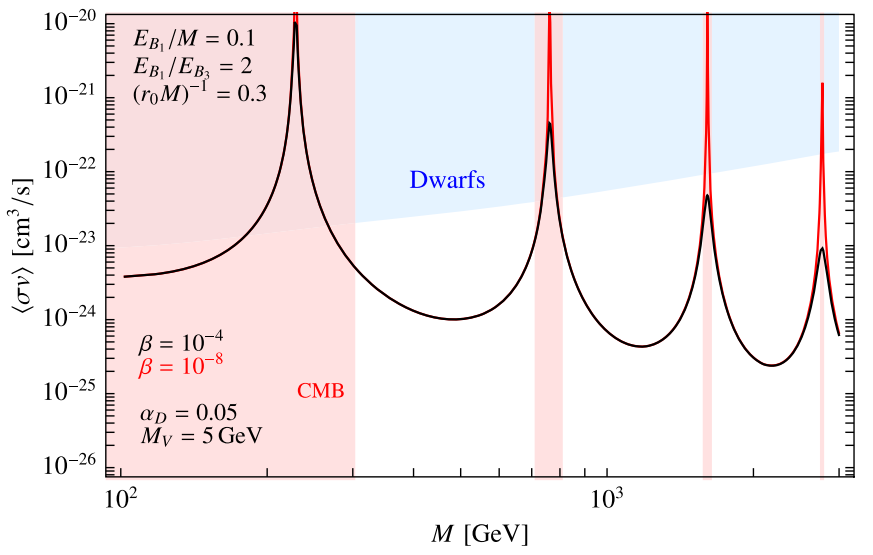

FIG. 3. Cross sections for dark deuterium formation $N_{+} N_{-} \rightarrow D_{1}+\gamma_{D}$ as a function of mass in models with SM-singlet composite DM and a dark photon. The light red regions are excluded by the CMB $\left(\beta \sim 10^{-8}\right)$; see Eq. (10). Left panel: Constraints for light DM and small binding energies and couplings, as dictated by the CMB constraints. Right panel: Constraints for intermediate DM masses and sizeable binding energies and couplings; indirect-detection constraints (light blue region) arise from diffuse $\gamma$-ray spectra from dwarf spheroidal galaxies as extracted from [22] for $10 \%$ branching fraction for $\gamma_{D} \rightarrow \tau \tau$ [21]). 


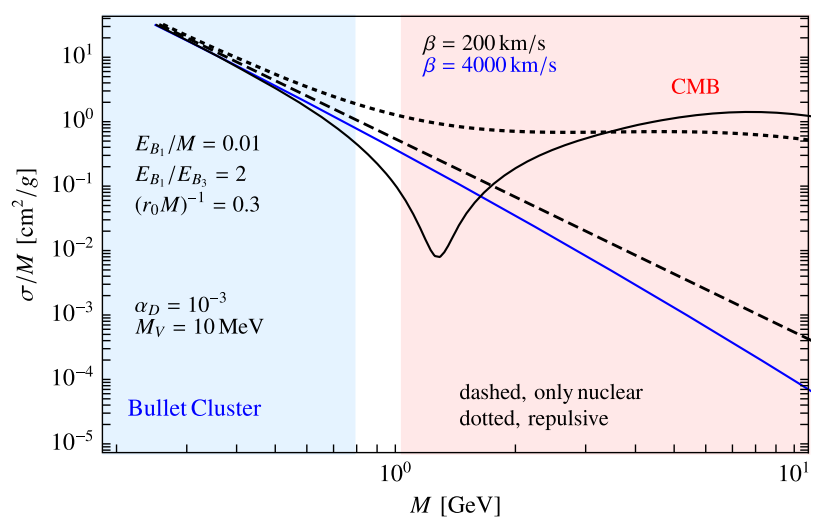

FIG. 4. Elastic cross section from DM self-interactions as a function of DM mass, for the allowed region of Fig. 3 (left). The bullet cluster constraint corresponds to $\beta \approx 4000 \mathrm{~km} / \mathrm{s}$, while $\beta \approx$ $200 \mathrm{~km} / \mathrm{s}$ is a velocity typically found in galactic cores [27].

is small; see [10]. Changing the parameters may result in dark deuterium, and possibly heavier dark nuclei, being synthesized primordially. This would reduce the indirectdetection rate, but could also potentially trigger processes such as dark tritium formation. Indeed, barring bottlenecks, light nuclear DM could produce a sizeable population of heavy dark nuclei $[25,26]$ resulting in novel phenomena that would merit detailed analysis.

Our framework will also be constrained by the size of DM self-interactions. These are particularly important for binding energies smaller than the mediator mass, where bound-state formation by dark photon emission is kinematically forbidden and the strong CMB bound no longer holds. The $s$-wave elastic cross section is given by $\sigma_{\mathrm{el}}=4 \pi / p^{2} \sin ^{2} \delta \approx 4 \pi a^{2} /\left(1+p^{2} a^{2}\right)$. We compute it by extracting phase shifts from solutions of the Schrödinger equation (4) using $e^{2 i \delta}=e^{-i p r_{\infty}}\left(u^{\prime}\left(r_{\infty}\right)+i p u\left(r_{\infty}\right)\right) / \sqrt{4 \pi}$; in different regions of parameter space these can be dominated either by the nuclear forces or by the longrange interactions. The cross section is strongly dependent on the character of the force mediated by the massive dark photon. For opposite-sign DM particles the potential is attractive and the rate displays resonance peaks where the cross section saturates to $\sigma_{\mathrm{el}} \sim 4 \pi / p^{2}$. For same-sign particles there is no resonance structure but the enhancement can still be sizeable. We illustrate this phenomenon in Fig. 4 where the vector boson mass has been tuned to yield a small region of dark matter parameter space which is consistent with both $\mathrm{CMB}$ and bullet cluster constraints [27]. Decreasing the binding energy relative to the mediator mass would open up this allowed region as bound-state formation becomes kinematically forbidden. Note that in some regions of parameter space the velocity dependence of the cross section allows the Bullet Cluster constraint to be satisfied, while simultaneously giving rise at lower velocities to a cross section $\sigma_{\mathrm{el}} / M \gtrsim \mathrm{cm}^{2} / \mathrm{g}$ that could explain observed small-scale properties of DM; see [28] for a review.

\section{SUMMARY AND OUTLOOK}

In this paper we studied the indirect-detection signal associated with the formation of bound states of DM, due to the emission of quanta with energy equal to the binding energy of the bound state. This can lead to a monochromatic photon line or diffuse $\gamma$-ray emission within reach of existing experiments such as FERMI. Deexcitation to the ground state could produce additional lines; a striking signature of bound-state formation that is easily distinguishable from annihilating DM.

This mechanism is particularly relevant for detection of asymmetric DM, which does not annihilate and would not typically give rise to a measurable indirect-detection signal. It is also relevant for thermal DM, where the photons emitted in bound-state formation would be complementary to the signal from direct annihilation of DM to photons.

We focused on magnetic-dipole interactions of (dark) nuclear DM in two simple and compelling scenarios. Despite the strongly coupled nature of the nuclear interactions the production cross section for bound states can be calculated at leading order in terms of the binding energies. Determining these in a strongly coupled $\mathrm{SU}(N)$ gauge theory is an interesting problem that merits further study, and could be solved on the lattice.

In this paper we have just skimmed the surface of the fascinating phenomenology of strongly coupled dark matter bound states. Similar effects can arise due to electric dipole interactions, for example, or emission of "dark pions." Furthermore, for large binding energies, emission of $W$ and $Z$ bosons may become kinematically allowed, leading to novel signatures.

\section{ACKNOWLEDGMENTS}

We thank Filippo Sala for useful comments. This work is supported by MIUR Grants No. PRIN 2017FMJFMW and No. 2017L5W2PT, Ente Cassa di Risparmio di Firenze and INFN Grant No. STRONG. 
[1] K. Petraki and R. R. Volkas, Int. J. Mod. Phys. A 28, 1330028 (2013).

[2] K. M. Zurek, Phys. Rep. 537, 91 (2014).

[3] L. Pearce and A. Kusenko, Phys. Rev. D 87, 123531 (2013).

[4] L. Pearce, K. Petraki, and A. Kusenko, Phys. Rev. D 91, 083532 (2015).

[5] W. Detmold, M. McCullough, and A. Pochinsky, Phys. Rev. D 90, 115013 (2014).

[6] A. Mitridate, M. Redi, J. Smirnov, and A. Strumia, J. Cosmol. Astropart. Phys. 05 (2017) 006.

[7] I. Baldes, M. Cirelli, P. Panci, K. Petraki, F. Sala, and M. Taoso, SciPost Phys. 4, 041 (2018).

[8] O. Antipin, M. Redi, A. Strumia, and E. Vigiani, J. High Energy Phys. 07 (2015) 039.

[9] G. D. Kribs and E. T. Neil, Int. J. Mod. Phys. A 31, 1643004 (2016).

[10] M. Redi and A. Tesi, J. High Energy Phys. 04 (2019) 108.

[11] H. A. Bethe and C. Longmire, Phys. Rev. 77, 647 (1950).

[12] D. B. Kaplan, M. J. Savage, and M. B. Wise, Phys. Lett. B 424, 390 (1998).

[13] M. J. Savage, K. A. Scaldeferri, and M. B. Wise, Nucl. Phys. A652, 273 (1999).

[14] D. B. Kaplan, arXiv:nucl-th/0510023.

[15] R. Mahbubani, M. Redi, and A. Tesi (to be published).
[16] J. Hisano, S. Matsumoto, M. Nagai, O. Saito, and M. Senami, Phys. Lett. B 646, 34 (2007).

[17] P. Asadi, M. Baumgart, P. J. Fitzpatrick, E. Krupczak, and T. R. Slatyer, J. Cosmol. Astropart. Phys. 02 (2017) 005.

[18] An approximate formula for the relation between the depth of a spherical well and the binding energy is $V_{a} / M \approx \pi^{2} /\left(4 r_{0}^{2} M^{2}\right)+2 \sqrt{E_{B_{a}} / M} /\left(r_{0} M\right)$.

[19] M. Ackermann et al. (Fermi-LAT Collaboration), Phys. Rev. D 91, 122002 (2015).

[20] H. Abdalla et al. (HESS Collaboration), J. Cosmol. Astropart. Phys. 11 (2018) 037.

[21] M. Cirelli, P. Panci, K. Petraki, F. Sala, and M. Taoso, J. Cosmol. Astropart. Phys. 05 (2017) 036.

[22] S. Profumo, F.S. Queiroz, J. Silk, and C. Siqueira, J. Cosmol. Astropart. Phys. 03 (2018) 010.

[23] T. R. Slatyer, N. Padmanabhan, and D. P. Finkbeiner, Phys. Rev. D 80, 043526 (2009).

[24] P. A. R. Ade et al. (Planck Collaboration), Astron. Astrophys. 594, A13 (2016).

[25] G. Krnjaic and K. Sigurdson, Phys. Lett. B 751, 464 (2015).

[26] E. Hardy, R. Lasenby, J. March-Russell, and S. M. West, J. High Energy Phys. 06 (2015) 011.

[27] S. Tulin and H.-B. Yu, Phys. Rep. 730, 1 (2018). 